Revue d'histoire de l'enfance « irrégulière »

Le Temps de l'histoire

$8 \mid 2006$

Le corps du délinquant

Philippe Tissié ou l'éducation physique au secours de la dégénérescence de la jeunesse française (1888-1935)

Jean Saint-Martin

(2) OpenEdition

Journals

Édition électronique

URL : http://journals.openedition.org/rhei/374

DOI : $10.4000 /$ rhei.374

ISBN : 978-2-7535-1646-5

ISSN : 1777-540X

Éditeur

Presses universitaires de Rennes

Édition imprimée

Date de publication : 15 novembre 2006

Pagination : 119-132

ISSN : 1287-2431

Référence électronique

Jean Saint-Martin, «Philippe Tissié ou l'éducation physique au secours de la dégénérescence de la jeunesse française $(1888-1935)$ », Revue d'histoire de l'enfance « irrégulière » [En ligne], 8 | 2006, mis en ligne le 01 décembre 2008, consulté le 03 décembre 2020. URL : http://journals.openedition.org/rhei/ 374 ; DOI : https://doi.org/10.4000/rhei.374 


\section{Philippe Tissié ou l'éducation physique au secours de la dégénérescence de la jeunesse française (1888-1938)}

\section{Introduction}

En reprenant, deux décennies après sa publication, l'article de M. Grosmolard édité en 1904 sur les Jeunes criminels en correction, ${ }^{(2)}$ le docteur Philippe Tissié tente de renforcer durant les années 1920 ses propositions en matière de rééducation de l'enfance irrégulière. L'état sanitaire de la France représente de ce point de vue un allié de circonstance à son discours sur la dégénérescence de la race au lendemain de la Grande Guerre. Pour cet auteur, en effet, les signes biologiques de la dégénérescence humaine ne doivent pas être les seuls analyseurs possibles, même s'ils sont largement présents en temps de crise. Depuis 1894, la gymnastique médicale rend, selon lui, « de bons services dans le traitement des maladies fonctionnelles du système nerveux et dans les maladies de dégénérescence ». ${ }^{(3)}$ Grâce à ses initiatives menées au sein de sa clinique de psycho-dynamie et de la société médicale de Pau, toutes deux créées en 1900, aux cours d'éducation physique qu'il dispense à l'école normale d'institutrices de Pau entre 1903 et 1913, mais aussi dans la maison de l'enfance de cette ville, Philippe Tissié, un des premiers neuropsychiatres de France, élabore une conception à la française d'une éducation physique permettant d'atteindre "la cérébration par la musculation ». ${ }^{(4)}$ Surnommé le Ling français en référence à l'inventeur de la méthode suédoise d'éducation physique, Per-Henrik Ling, il s'intéresse progressivement aux délinquants au nom de la défense de la "Terre de France ", en considérant que la méthode franco-suédoise d'EP participe du processus de normalisation et de redressement des jeunes criminels, qu'il qualifie de "paresseux et d'impulsifs ». ${ }^{(5)}$

\section{Jean Saint- Martin $^{(1)}$}

(1) Maître de conféren-

ce, Centre de recherche

d'innovation sur le sport (CRIF, EA 647), Lyon I.

(2) M. Grosmolard,

"Les jeunes criminels en correction ", Archives d'anthropologie criminelle, de criminologie, et de psychologie normale et pathologique, t. 19, 1904, p. 341 455. M. Grosmolard dirige, au tournant du siècle, une colonie pénitentiaire.

(3) Philippe Tissié, «Un cas d'instabilité mentale avec impulsions morbides traité par la gymnastique médicale ", Archives cliniques de la faculté de Bordeaux, mai 1894, p. 244. 
(4) Philippe Tissié,

"L'éducation physique et le patriotisme chez les jeunes criminels en correction ", La Revue des jeux scolaires et d'hygiène sociale, 1926, p. 19.

(5) Ibid., p. 15.

(6) Sur l'histoire des rapports entre l'éducation physique et l'hygiène, se reporter notamment à Georges Vigarello, Le corps redressé, Paris, J.-P. Delarge, 1978, 399 p.

(7) Jacques Léonard, Archives du corps. La santé au XIXème siècle, Rennes, Ouest-France université, 1986, 331 p.

(8) B.-A. Morel, Traité des dégénérescences physiques, intellectuelles et morales de l'espèce humaine et des causes qui produisent ces variétés maladives, Paris, Baillière, 1857, $700 \mathrm{p}$.
(9) Il s'agit du titre de sa thèse de médecine soutenue en 1887 . Son directeur de recherche n'est autre que le Pr. Pitres. Sur cette question, se reporter à Gilles Lecocq, "Maladies de la volonté... et "Voyageurs infatigables" ", in Christian Pociello, Entre le social et le vital, Grenoble, PUG, 2004, p. 113-123.

(10) Philippe Tissié, La fatigue et l'entraînement physique, Paris, Alcan, 1897, 336 p.

(11) À titre d'exemple, se reporter à l'article publié dans les actes du congrès de médecins aliénistes et neurologistes de France de 1895 :

«Traitement des phobies par la suggestion et la gymnastique médicale ", p. $452-461$.

(12) Philippe Tissié, L'éducation physique et la race : santé - travail longévité, Paris, Flammarion, 1919.

\section{Rééduquer les "dégénérés"}

Depuis la création de la Ligue girondine d'éducation physique (LGEP) en octobre 1888, Philippe Tissié a toujours été attentif à la reconstruction d'une jeunesse saine et vigoureuse. ${ }^{(6)}$ Fidèle aux idéaux républicains, il n’envisage pas de reconstruction nationale sans un enseignement obligatoire de l'éducation physique à l'école. Pendant un demi-siècle, il œuvre au développement et à l'institutionnalisation d'une éducation physique eugénique, dont l'enseignement est devenu obligatoire à l'école de 6 à 13 ans avec la loi George du 27 janvier 1880. En transformant, au tournant du siècle, la LGEP en Ligue française d'éducation physique (LFEP), Philippe Tissié tente de venir au secours d'une santé nationale jugée encore déficiente par les médecins de cette époque. ${ }^{(7)}$ Sa contribution demeure cependant originale, car Philippe Tissié réaffirme l'importance des caractéristiques psychologique et mentale des dégénérescences. ${ }^{(8)}$

Ayant mené de nombreux travaux scientifiques au sein de l'Académie de médecine de Bordeaux, il envisage de soigner aussi bien "les aliénés voyageurs ", ${ }^{(9)}$ que les Françaises et Français présentant une fatigue chronique, plus communément appelée surmenage. ${ }^{(10)}$ Quelle que soit la pathologie envisagée, Philippe Tissié multiplie les propositions pour faire reculer la maladie et rendre ainsi leur pouvoir d'agir aux citoyennes et citoyens attachés à leur devoir de santé.(11)

Juste avant la première guerre mondiale, une nouvelle opportunité se présente. En 1913, grâce à l'enquête de Alfred de Tarde et Henri Massis, intitulée Les jeunes gens d'aujourd'hui, que ces deux auteurs publient sous le pseudonyme d'Agathon, Philippe Tissié s'engage résolument dans l'enseignement aux générations futures des valeurs traditionnelles telles que le goût héroï- 
que, le culte de la tradition classique et celui du renouveau moral. Cet engagement est ponctué par la publication, en 1919, d'un ouvrage écrit en hommage aux Poilus de la Grande Guerre, L'Éducation physique et la race. ${ }^{(12)}$ Pour persuader l'opinion publique française, Philippe Tissié choisit de regrouper tous les malades atteints de difformités physiques et psychologiques sous le vocable de dégénérés.

Il considère en effet l'enfant délinquant " comme un dégénéré, à évolution psychique ralentie, déviée ou déséquilibrée ", ${ }^{(13)}$ en qui prime le goût de ce qui attire le regard, de l'éphémère et du paraître. Au lendemain de 1914-1918, il est d'autant plus urgent de redresser ces jeunes instables que leur système de valeurs ne correspond pas aux mentalités françaises. Selon lui, "goûts de lutte, de déprédation, solidarité partisane, sociétés secrètes " ${ }^{(14)}$ sont à proscrire dans une France qui cherche à recouvrer son prestige politique et culturel et pour qui « la guerre a porté un coup très dur aux valeurs qui constituent les assises de la civilisation occidentale ». ${ }^{(15)}$ Au sein de la LFEP, il souhaite " fortifier des volontés, des intelligences et des caractères, élargir l'idéal de la jeunesse et éduquer le sentiment de la responsabilité individuelle par les exercices physiques et les jeux sportifs dans l'action collective ". ${ }^{(16)}$ Alors que le taux de criminalité s'élève au lendemain de la Grande Guerre à 9,4 pour 100.000 habitants, ${ }^{(17)}$ Philippe Tissié reprend à sa manière le credo de Victor Hugo, selon lequel il suffirait d'ouvrir des écoles pour fermer des prisons, bref éduquer et prévenir avant d'enfermer. Selon lui, en enseignant le système de gymnastique franco-suédois, qui répond « à tous les desiderata de la thérapeutique par le mouvement physique ", ${ }^{(18)}$ il est possible de rééduquer les jeunes délinquants en offrant une seconde chance, celle de participer à la reconstruction nationale. Il espère ainsi pouvoir constituer, " par une bonne hygiène sociale, des machines humaines à rendement puissant et prolongé ". ${ }^{(19)}$

Pour convaincre, il adresse de nombreuses lettres aux parlementaires et responsables du pouvoir exécutif. ${ }^{(20)}$ En faisant la promotion des techniques corporelles issues de données scientifiques et finalisées par des contingences sociales, Philippe Tissié entend plus que jamais rendre "à la jeunesse française la force de bien penser et de bien agir, la souplesse du corps et de celle de l'esprit ; la gaieté qui disparaît et le rire du bon vieux temps ».(21) Grâce à une méthode sûre, une valeur propre, une volonté( ${ }^{(22)}$ tenace, Philippe Tissié
(13) Philippe Tissié,

"L'éducation physique et le patriotisme... ", op. cit., p. 18.

(14) Ibid.

(15) Pierre Milza, Serge Berstein, Histoire du XXème siècle, I900-1945, la fin du "monde européen", Paris, Hatier, 1993 , p. 110.

(16) Philippe Tissié, L'éducation physique au point de vue historique, scientifique, technique, critique, pratique et esthétique, Paris, Larousse, 1901 , p. 6.

(17) Théodore Zeldin, Histoire des passions françaises, I848-1945. Anxiété et hypocrisie, tome 5, Paris, Seuil, 1979, p. 164.

(18) Philippe Tissié, L'éducation physique au point de vue historique..., op. cit., p. 123. 
(19) La Revue des jeux scolaires..., janvier 1906.

(20) En 1926, il s'adresse en personne à

Raymond Poincaré. Deux ans plus tard, c'est au tour d'Édouard Herriot et des présidents des commissions de la Chambre des députés.

(21) Discours prononcé par le Dr Philippe Tissié le 19 décembre 1888 lors de la fondation de la LGEP. Archives de la FFEPGV, dossier $\mathrm{n}^{\circ} 1$, document $4 \mathrm{c}$.

(22) En mettant au cour de sa conception d'éducation physique la notion de volonté, Philippe Tissié s'inspire des travaux de T. Ribot et confirme l'influence de la chaire de psychologie expérimentale et comparée du Collège de France. Sur cette question, se reporter à Gilles Lecocq, «De Théodule Ribot à Ferdinand Buisson ", in
Christian Pociello, Entre le social et le vital, Grenoble, PUG, 2004, p. 95-112.

(23) Il emploie cette expression dès 1908 dans La Revue des jeux scolaires et d'hygiène sociale.

(24) Philippe Tissié, «L'évolution de l'éducation physique en France 1903-1933 ", La Revue des jeux scolaires et d'hygiène sociale, 1933, p. 76.

(25) Henri Thulié, Le dressage des jeunes dégénérés ou orthophrénopédie, Paris, Alcan, 1900, 678 p.

(26) Ibid., p. 178.

(27) Serge Fauché, Techniques du corps et traitements de l'esprit aux XVIIIème et XIXème siècles, Paris, L'Harmattan, 1997, 269 p. lutte contre les « déchets sociaux ", ${ }^{(23)}$ " en spiritualisant le mouvement par la réflexion et en muscularisant la pensée par le réflexe discipliné et éduqué ». ${ }^{(24)}$ Il conçoit tout simplement sa méthode de gymnastique comme un élément à part entière de la science du redressement de l'affect et de la raison, l'orthophrénopédie. ${ }^{(25)}$ L'enjeu éducatif central consiste à contrôler tous les gestes qui échappent à leur auteur et qui peuvent être à l'origine d'internement, voire d'emprisonnement s'il s'agit de gestes délictueux. Par un enseignement rigoureux, la gymnastique francosuédoise limite les gestes impulsifs, involontaires, inconscients, irrépressibles et incontrôlés. Par une segmentation rationnelle des mouvements et une simultanéité parfaite, la psycho-dynamie de Philippe Tissié éduque tout autant qu'elle instruit les individus. Pour lui, l'exercice physique force l'attention, fait travailler le cerveau, suscite l'initiative et la volonté. Il assouplit le caractère en imposant l'habitude d'exécuter des ordres commandés, établissant ainsi «par la continuité de sa pratique, un réel réflexe de l'obéissance ". ${ }^{(2)}$

La méthode franco-suédoise poursuit ainsi trois finalités : développer la santé, améliorer la race, servir la patrie. En apprenant à maîtriser et à perfectionner la pensée, en inculquant aux jeunes déviants les valeurs du travail et de la volonté, Philippe Tissié souhaite participer au redressement social de la France et représente les gymnastiques de formation qui luttent depuis la fin du XIXème siècle contre les penchants criminels des aliénés ou des dégénérés. ${ }^{(27)}$ Cette gymnastique franco-suédoise est composée successivement d'une gymnastique de formation et de constitution, sorte de grammaire gestuelle fondamentale, qui développe harmonieusement le corps, depuis celui de l'enfant jusqu'à celui du soldat, puis d'une gymnastique d'application 
et d'adaptation sociale, qui tantôt répare, rétablit les forces pour le relèvement et la reprise du " moi » (gymnastique thérapeutique et clinique), tantôt ennoblit le " moi » en enseignant des gestes eurythmiques, des attitudes plastiques (gymnastique esthétique et artistique).

Souhaitant transformer les jeunes criminels en hommes de caractère, sa gymnastique du corps et de l'esprit cherche à amplifier la "volonté de vouloir ». Les exercices corporels sont avant tout justifiés par leurs fonctions disciplinaires et leur pouvoir de fabriquer la volition, une qualité psychologique qui spécifie, selon lui, la condition humaine.

\section{Transformer les " passifs " en " affirmatifs"}

Lorsque Philippe Tissié présente, en 1926, l'ouvrage de Grosmolard de 1904, l'expérience accumulée depuis un quart de siècle au sein de sa clinique de psycho-dynamie ${ }^{(28)}$ lui permet d'avoir une vision déjà très précise de l'enfance délinquante. Principale victime de la Grande Guerre, l'enfant peut, à ses yeux, être sauvé d'un destin malheureux. Grâce à ses expériences paloises, Philippe Tissié propose une taxonomie de l'être humain : les passifs, les affectifs et les affirmatifs.

Les passifs constituent les êtres les plus faibles par nature. Ils sont incapables de prendre des initiatives et sont généralement à la merci des autres. Selon Philippe Tissié, le passif agit sous le commandement, il suit, il s'arrête devant la responsabilité ou la difficulté. Ces suivistes dépersonnalisés représentent pour lui des cas pathologiques de première importance pouvant être guéris par une saine rééducation physique et mentale. Il s'agit en outre ici de bannir les techniques traditionnelles d'apprentissage qui parfois reproduisent des croyances contre lesquelles Philippe Tissié s'insurge. En effet, les jeunes détenus, ayant un respect exagéré de la force, doivent être persuadés que la puissance du muscle ne justifie en aucun cas la domination du plus fort. Généralement incapable d'initiative, le passif ne cherche pas à comprendre ni à modifier le cours de sa vie. Pour le solliciter, Philippe Tissié utilise le « je veux ", qui induit un ordre, une suggestion impérative permettant à ces « instinctifs et primitifs " ${ }^{(29)}$ d'entrevoir une émancipation sous tutelle, c'est-à-dire contrôlée par l'adulte éducateur et non par un autre délinquant exerçant sur lui une tyrannie.

(28) Philippe Tissié présente officiellement la psycho-dynamie en 1901, dans son opuscule publié à l'imprimerie Gounouilhou de Bordeaux, Exercice physique au point de vue thérapeutique.

(29) Philippe Tissié, "L'éducation physique et le patriotisme..." ", op. cit., p. 15. 
(30) Philippe Tissié,

L'éducation physique et la race..., op. cit., p. 140.

(31) Philippe Tissié, ibid., p. 139.

(32) Philippe Tissié, "L'éducation physique et le patriotisme..." ", op. cit., p. 16.
Les affectifs sont, quant à eux, des personnes qui doutent d'elles-mêmes et qui parfois s'ignorent. La plupart du temps, ils n'osent agir par crainte d'impuissance et demeurent à la merci de multiples dépendances. Influençables à souhait, une gymnastique médicale bien adaptée permet de leur redonner confiance et d'en faire des gens bons pour être utiles. Ils s'attachent généralement à ceux qu'ils sentent plus forts qu'eux. Selon Philippe Tissié, les affectifs fournissent des " hypocrites et des révoltés ", et constituent le plus souvent les criminels qui accusent le milieu social, la famille ou la société d'avoir «brutalisé leur sensibilité enfantine et leur affectivité native sinon naïve ». ${ }^{(30)}$ Pour lui, beaucoup de criminels sont en effet des affectifs mal suggestionnés qui extériorisent leur révolte par des actes délictueux. Pour solliciter les affectifs, Philippe Tissié utilise le " tu peux ", qui représente une suggestion persuasive en jouant sur la volonté du détenu, prêt à accepter de travailler de nouveau. Même si cette habitude disparaît très rapidement dans le milieu carcéral, la bonne pédagogie de redressement prônée par Philippe Tissié consiste à ennoblir le travail, quitte à jouer sur la naïveté des jeunes délinquants.

Enfin, les affirmatifs sont le plus souvent des indépendants, des égoïstes qui, bien canalisés, réalisent de véritables prouesses physiques et mentales. Attirés par l'effort, ils recherchent sans cesse la difficulté et repoussent leurs limites. L'action devient synonyme de plaisir à condition qu'elle soit finalisée vers l'intérêt collectif. Selon Philippe Tissié, l'affirmatif est né pour commander, et « un peuple chez lequel l'éducation tendrait à former de tels caractères serait appelé aux plus hautes destinées ». ${ }^{(31)}$ L'affirmatif déviant, quant à lui, s'il est " généreux et dévoué à ses amis, il est vindicatif et cruel pour ses ennemis. La vie d'un camarade ou d'un surveillant ne compte pas ». ${ }^{(32)}$ Les prisons semblent garnies d'affirmatifs déviants, l'impulsivité des jeunes détenus étant à l'origine de cette situation. Pour solliciter les affirmatifs, Philippe Tissié utilise le "tu ne peux pas ", suggestion négative devant provoquer une action affirmative par contre-suggestion. Philippe Tissié fait appel ici au sursaut d'orgueil, qu'il s'agit cependant de canaliser par une gymnastique frénatrice des impulsions engendrées par le défi proposé.

Cet essai de caractérologie, sans précédent dans le monde de l'éducation physique, est destiné à aider les éducateurs dans leurs tâches quotidiennes, qu'il s'agisse des instituteurs ou des surveillants. La conscience de Philippe 
Tissié est si développée qu'il distingue les notions d'éducation et d'élevage, la seconde devant laisser la priorité à la première :

"L'éducation est synonyme de méthode réfléchie, prudente et opportune, d'action lente et douce, de respect de chaque sujet et de souci de son développement progressif par des sentiments affectifs d'ordre supérieur où le cœur domine l'esprit, où la volonté est persuasive, où la solidarité faite d'estime réciproque est comme une appétence de l'âme au dévouement. L'éducation est le soutien des faibles par la non-spécialisation, sa devise est la grande devise humaine : aimez-vous les uns les autres. "(33)

Entre les deux guerres mondiales, Philippe Tissié peaufine son essai de caractérologie. Pour lui, les individus doivent être davantage classés d'après leur capacité d'adaptation au milieu social. Seul le caractère, qui représente l'agent d'adaptation prioritaire, permet d'affiner cette discrimination en trois grandes catégories. Sur le plan scientifique, on note ici l'influence déterminante de Pierre Janet, disciple et successeur de Théodule Ribot au Collège de France. Auteur de l'article traitant de l'attention volontaire dans l'éducation physique, publié dans le rapport de Philippe Tissié de 1901, Pierre Janet donne à la psycho-dynamie ses lettres de noblesse. Il permet en fait de démontrer que le mouvement musculaire par lui-même ne représente qu'une importance secondaire et qu'il importe avant tout que chaque sujet soit réactif sur le plan psychique. Au lendemain de la Grande Guerre, l'éducation des plus faibles sonne donc comme une priorité dans l'œuvre éducatrice de Philippe Tissié, car le devenir d'une race ne saurait dépendre exclusivement des plus forts. Apprendre à être sain pour être bon devient un des apophtegmes les plus connus de Philippe Tissié. La force, pourtant si " prisée par le détenu ", ${ }^{(34)}$ ne suffit pas, encore faut-il la mettre au service de la collectivité. Pour le Ling français, l'enjeu éducatif est essentiel pour les jeunes délinquants. Pour ces impulsifs, "il fait bon d'être [encore] plus bon " ${ }^{(35)}$ grâce à un enseignement rigoureux permettant d'anticiper les comportements déviants. Selon lui, la gymnastique franco-suédoise s'apparente dans ces conditions à un médicament de salubrité nationale. Les propos tenus en 1926 en témoignent :

"Pour ce qui est des criminels en correction, il serait intéressant de les soumettre à une éducation analytique frénatrice des impulsions que les sports favorisent quand ils ne les provoquent pas. "(36)

(33) Philippe Tissié,

"Élevage et éducation",

La Revue des jeux scolaires et d'hygiène sociale, 1895 , p. 17.

(34) Philippe Tissié,

« L'éducation physique et le patriotisme...", op. cit., p. 15.

(35) Philippe Tissié, "Il faut opter ", La Revue des jeux scolaires et d'hygiène sociale, 1933, p. 33.

(36) Philippe Tissié, "L'éducation physique et le patriotisme...", op. cit., p. 19. 
(37) Philippe Tissié, L'éducation rationnelle, Paris, Alcan, 1922, p. 12-13.

(38) Emmanuel Decaux, « La ruée vers l'or ", dans Olivier Barrot et Pascal Ory, Entre-deuxguerres, la création française I9I9-I939, Paris, François Bourin, 1990, p. 229-251.

(39) Philippe Tissié, L'éducation rationnelle, op. cit., p. 56.

(40) Lieutenant-colonel Coste, L'éducation physique en France, ce qu'elle est, ce qu'elle devrait être, Paris, C. Lavauzelle, 1907, p. 59.
Pour lui, la gymnastique franco-suédoise doit les rééduquer le plus rapidement possible :

"La génération de la guerre est indisciplinée. L'esprit de soumission de l'enfant a été atténué par l'absence prolongée du père et par les mauvaises habitudes de liberté prises au foyer, privé de son chef. »

"La génération enfantée pendant la guerre offre des symptômes nerveux pathologiques inquiétants, d'impulsions violentes, d'émotivité outrée, de précocité intellectuelle surprenante, d'instincts dégénérés. Il faut la tonifier et la traiter par une éducation médico-pédagogique dans laquelle le mouvement physique discipliné de la gymnastique analytique aura de bons effets, car discipliner les muscles, c'est discipliner la pensée, et, par cette discipline frénatrice même, inhiber les impulsions morbides. " ${ }^{(37)}$

En disciplinant la pensée par les muscles, la conception suédiste de l'éducation physique ne sert pas seulement à faire de "bons moteurs humains ", elle contribue à la régénération morale de la race. La gymnastique analytique de formation se donne ainsi pour principale tâche de former des êtres humains dont le civisme, la bravoure, rappellent le modèle des Poilus. Pour Philippe Tissié, la jeunesse de l'immédiat après-guerre, qui découvre notamment Charlie Chaplin lors de sa tournée européenne de 1921 pour le lancement du Kid, ${ }^{(38)}$ a besoin d'être reprise en main. Son adhésion aveugle aux valeurs d'un américanisme triomphant lui déplaît. L'heure est donc aux sacrifices, aux efforts et non à l'amusement. Tout doit être entrepris pour éloigner des bals, des cabarets, des lieux de débauche, les jeunes et plus particulièrement les délinquants. Chacun doit en effet accomplir de réels efforts pour retrouver la plénitude de son rendement et permettre à la France de récupérer sa place et son prestige sur les échiquiers politiques, économiques et financiers européens. Véritable panacée pédagogique et thérapeutique, la méthode franco-suédoise d'éducation corporelle est à la fois " pédagogique et hygiénique pour la formation du moi, athlétique et militaire pour l'affirmation du moi, médicale et thérapeutique pour la réparation du moi, et sociale et économique pour le plus grand rendement énergétique du moi ». ${ }^{(39)}$ La méthode suédoise, revue et corrigée par Philippe Tissié, s'annonce donc comme un excellent moyen pour la discipline du geste, " forme première de la discipline de la raison, de l'obéissance à la loi ». ${ }^{(40)}$ Dans ces conditions, l'homme sans caractère est soumis à toutes les 
fluctuations de l'existence, "c'est un navire à la dérive sans mâture, sans gouvernail, sans boussole. [...] L'homme de caractère s'étant fixé un but le poursuit sans jamais dévier ; ignorant les compromissions, il accomplit son devoir, au jour le jour, en toute simplicité, en toute sincérité, quoi qu’il arrive, utile d'abord, heureux ensuite ". ${ }^{(41)}$ Seule la devise Fortitudo mea civium fides ${ }^{(42)}$ peut sauver la France d'une dégénérescence sociale et géopolitique.

Malgré tous ces avantages, les craintes des suédistes se confirment durant les années folles. "L'ardeur sportive " pousse notamment les jeunes délinquants à s'intéresser davantage aux joies du sport, aux multiples attraits des compétitions, qu'aux bienfaits sanitaires de cette gymnastique paramédicale. L'hédonisme des pratiques sportives les séduit de plus en plus. Ce phénomène embrasse toute l'Europe où l'on réclame massivement des pratiques basées sur les principes des jeux et/ou des compétitions sportives. Dans ce contexte, « avec le sport, la France donne des signes incontestables de joyeuse modernité ».(43)

\section{Le sens de l'effort utile}

La France de l'entre-deux-guerres doit promouvoir l'esprit de sacrifice, l'esprit d'effort, afin de se "rendre utile ", ${ }^{(44)}$ car " tout homme, qui ne peut faire effort utile, est vaincu d'avance ; toute société pour laquelle l'effort est pénible est une société appelée à disparaître. L'homme n'est pas fait pour se reposer mais pour travailler et pour faire effort ". ${ }^{(4)}$ Dans l'ouvrage dédié aux soldats français de 1914-1918, L'éducation physique et la race, Philippe Tissié est on ne peut plus explicite :

«En France, on ne sait pas s'habituer à un principe, à une règle. [...] L'EP [sic], branche la plus importante de l'hygiène, est avant tout un devoir à accomplir, et non un acte instinctif à commettre. En cela, l'EP est une morale. Sans la discipline, l'EP est sacrifiée ; avec la discipline, elle est sanctifiée. [...] La discipline implique la confiance, la confiance n'est conquise que par la compétence. Notre démocratie a tout à gagner à être guidée par des compétences. Créer des compétences, c'est élever le niveau moral de la démocratie, c'est la rendre moins vaniteuse, moins envieuse, moins jalouse ; vanité, envie, jalousie sont les filles de l'incompétence. " (46)

Opposé à la loi du moindre effort, il estime qu'un peuple ne vaut que par l'enfant ; " le problème à résoudre est celui-ci : créer chez l'enfant des ten-
(41) Philippe Tissié,

L'homme de demain, l'éducation physique en France, rapport présenté au congrès international d'expansion économique mondiale, Mons, 1905, section 1, enseignement, p. 11.

(42) La confiance des citoyens est ma force.

(43) Jean-Pierre Rioux, " L'ardeur sportive à la Belle Époque ", L'Histoire, $\mathrm{n}^{\circ} 14$, juillet-août 1979 , p. 78.

(44) Philippe Tissié, "La peur et le courage de vivre ", La Revue des jeux scolaires et d'hygiène sociale, 1933, p. 101.

(45) Philippe Tissié, L'éducation physique au point de vue historique..., op. cit., p. 16.

(46) Philippe Tissié, L'éducation physique et la race..., op. cit., p. 332. 
(47) Philippe Tissié, L'homme de demain..., op. cit., p. 12.

(48) Ibid., p. 5.

(49) Ibid.

(50) Philippe Tissié, « La maison de l'enfant à Pau ", La Revue des jeux scolaires et d'hygiène sociale, 1920, p. 164.

(51) «Les hommes heureux restent souples, agiles, ont le teint frais, la physionomie souriante et l'œil vif ; les hommes tristes se traînent alourdis et pâles, l'œil morne, la physionomie atone, le rictus contracté en flexion ", ibid., p. 6.

(52) Ibid.

(53) Philippe Tissié, L'homme de demain..., op. cit., p. 7.

(54) Philippe Tissié, L'éducation physique et la race..., op. cit., p. 43. dances vers le sens de l'effort utile, par le développement du caractère, dans la joie de vivre ". ${ }^{(47)}$ Quinze ans après le congrès international d'expansion économique de Mons de 1905, ses propos sont encore d'actualité lorsqu'il réclame un " homme pratique et fort, aimant la lutte pour la lutte et l'effort pour l'effort ". ${ }^{(48)} \mathrm{Il}$ s'agit en effet de " ne plus se payer de mots, mais d'envisager les actes ; de voir sans cesse la réalité à travers le rêve ; de ne se laisser jamais bercer par de chimériques illusions ; de rester toujours maître de soimême ; d'être généreux et bon ; volontaire et doux, conciliant avec ses semblables ». ${ }^{(49)}$

À vrai dire, ce discours a déjà fait ses preuves. Philippe Tissié s'appuie sur une œuvre éducative de la maison de l'enfant de Pau, ancien dispensaire de la Croix-Rouge des femmes de France, qui accueille des enfants déshérités âgés de 6 à 13 ans. À ces filles et fils de manœuvres espagnols et français, à ces pupilles de la Nation et parfois même à des arriérés mentaux des classes primaires, Philippe Tissié dispense durant deux heures hebdomadaires sa gymnastique éducative, dans le but de créer " une petite république de petits bonshommes dont les jugements sont sûrs ». ${ }^{(50)}$ Il s'agit ici de développer le sens de l'effort utile, en même temps que l'action et l'affirmation de l'individualité. Pour Philippe Tissié, le jeu procure la joie de vivre qui, à son tour, devient une force à part entière. Et l'auteur de comparer le " geste de la joie ", qui augmente la circulation sanguine, à la tristesse qui contracte, amplifie l'égoïsme de chacun. ${ }^{(51)}$ " Le geste de la joie » est donc bel et bien le " geste mondial ». ${ }^{(52)}$ Afin de convaincre, il introduit la référence à Tarde, qui considère la joie comme le fait « d'être et d'agir ensemble pour sympathiser, pour fraterniser. Qui dit société, dit joie ; la joie est la fleur naturelle de la sociabilité ». ${ }^{(53)}$

L'enjeu de sa gymnastique franco-suédoise consiste donc à apprendre l'utilité et la gratuité de l'effort individuel et collectif, parce qu'une nation " qui se satisfait du nécessaire est une nation paresseuse ". ${ }^{(54)}$ À tel point que l'un des slogans du discours du Ling français pourrait être « faire effort et vouloir se rendre utile " ${ }^{(55)}$ :

"Tout homme qui ne peut faire effort utile est vaincu d'avance ; toute société pour laquelle l'effort est pénible est une société appelée à disparaître. L'homme n'est pas fait pour se reposer mais pour travailler et pour faire effort, c'est pourquoi il doit gagner son pain à la sueur de son front ; 
ce devoir ne saurait être considéré comme une punition, mais, bien au

(55) Philippe Tissié, contraire, comme une haute récompense de la nature qui a fait son élu de l'homme fort, produisant par un travail constant. "(56)

L'auteur renchérit cinq ans plus tard :

"Il est nécessaire d'affiner tout particulièrement chez les jeunes générations le sens de l'effort utile en vue d'une plus grande expansion de la patrie dans les compétitions âpres, complexes et nombreuses qui se partagent le monde actuellement. " (57)

Au lendemain de la Grande Guerre, ce discours apparaît tout à fait pertinent. Philippe Tissié rappelle cet enjeu aux instituteurs, auxquels il dédie son ouvrage L'éducation rationnelle, de 1922 :

«Vivre consiste à agir [...]. Le problème social à résoudre consiste à créer chez l'enfant des tendances à l'action dans l'effort utile par le mouvement ordonné et discipliné, par une éducation physique rationnelle, en vue de la formation et du développement du caractère, dans la joie de vivre qui est une force, en toute plénitude de production et de rendement économique social. " ${ }^{(58)}$

Cet éloge de l'effort construit et utile, de la volonté et de l'abnégation, ce mépris de la douleur seront repris sous le régime de Vichy. Nous le voyons, l'enjeu essentiel est bel et bien de discipliner et de responsabiliser la jeunesse de France, en éduquant son âme grâce à une juste éducation physique. L'œuvre de Tissié poursuit donc des finalités républicaines, où la liberté de la nation dépend de la responsabilité de ses citoyens, où l'éducation du caractère permet à un peuple affaibli de recouvrer sa solidarité. Philippe Tissié prône finalement un apprentissage de l'exercice de la citoyenneté en et par une éducation physique construite et rationnelle, y compris pour les délinquants. Utile à la société, utile à la démocratie qui lui confere des droits et des devoirs, tout jeune de France doit faire preuve de sérieux en n'accordant pas plus de place qu'ils n'en méritent aux jeux et à leurs formes plus élaborées, les sports. En alternant scientifiquement les apprentissages de techniques corporelles, véritable grammaire du geste, et les jeux, moments privilégiés de l'expression de la personnalité de chacun, la gymnastique éducative de Philippe Tissié transmet les vertus de la souffrance et du plaisir qui en résulte. Son éducation physique vise donc à marteler les caractères des jeunes gens et des jeunes filles de France, considérée comme le levain de la race, car :
« La peur et le courage de vivre ", op. cit., p. 101.

(56) Philippe Tissié, L'éducation physique au point de vue historique..., op. cit., p. 16.

(57) Philippe Tissié, " Notre programme", La Revue des jeux scolaires et d'hygiène sociale, 1906, p. 3.

(58) Philippe Tissié, L'éducation rationnelle, op. cit., p. 200. 
(59) Philippe Tissié, L'homme de demain..., op. cit., p. 11.

(60) Philippe Tissié, L'éducation physique et la race..., op. cit., p. 272.

(61) Philippe Tissié, L'éducation rationnelle, op. cit., p. XI.

(62) Philippe Tissié, L'éducation physique et la race..., op. cit., p. 283.

(63) Philippe Tissié, "Le sport tabou ", La Revue des jeux scolaires et d'hygiène sociale, 1932, p. 5.

(64) Jean Saint-Martin, « La force nationale par le sport. Analyse géopolitique de l'éducation physique française entre les deux guerres mondiales (1918-1939) ", in Gigliola Gori, Thierry Terret, [coord.], Sport et éducation, Sankt Augustin, Academia Verlag, 2005, p. $272-279$
"Le caractère est une des grandes manifestations des forces psychiques humaines. Il est fait de jugement, de raison, d'intelligence, de volonté et de beauté. [...] L'homme sans caractère est soumis à toutes les fluctuations de l'existence, c'est un navire à la dérive sans mâture, sans gouvernail, sans boussole. L'homme sans caractère traite souvent de mauvais caractère celui qui, en possédant un, l'affirme. Il confond l'entêtement avec la volonté. [...] L'homme de caractère s'étant fixé un but le poursuit sans jamais dévier ; ignorant les compromissions, il accomplit son devoir, au jour le jour, en toute simplicité, en toute sincérité, quoi qu'il arrive, utile d'abord, heureux ensuite. "(59)

En 1919, il explique une nouvelle fois qu'il est urgent de dompter la foule : "Méthode, ordre, discipline, compétence, responsabilité, hiérarchie, tout cela est inconnu de la foule désordonnée et impulsive à mentalité animalienne ; tout cela doit être imposé à la foule mieux ordonnée par l'école éducatrice. " ${ }^{(60)}$

Patriote sans être chauvin, militariste à sa manière mais non belliqueux, pacifiste et humaniste, Philippe Tissié combat ainsi la loi du moindre effort et reste d'autant plus vigilant qu'il adhère aux propos de Georges Clemenceau, de 1919 : "gagner la paix sera encore plus difficile ". S’il prône une éducation physique et morale de toute la jeunesse française, y compris la jeunesse délinquante, il n'en demande pas moins que la "Terre de France » reste l'arme au pied, attentive aux évolutions géopolitiques. Seule compte « l'assurance dans l'avenir, par le travail réparateur et fécond, dans la joie de vivre, sans avoir à redouter de nouveaux conflits armés ". ${ }^{(61)}$

Il s'agit donc de mettre fin au mal dont souffre la France, la paresse qui s'applique systématiquement à détruire les bonnes volontés de la LFEP. ${ }^{(62)} \mathrm{Au}$ cours des années 1930, Philippe Tissié précise les déviances du sport moderne qui peuvent amplifier les comportements déviants des jeunes détenus. Cette nouvelle mise en garde est essentielle à ses yeux, car elle permet de dissocier la re-création, mission beaucoup plus noble poursuivie par Philippe Tissié, de la récréation, objectif essentiel des sociétés sportives qui forment non plus des citoyens, mais des "ludomanes impulsifs" :

"De même que la discipline grammaticale des mots assure celle de la phrase et du discours ; que celle de la note gammée du solfege assure celle de 
l'exécution de la partition musicale, la discipline du muscle, par la gymnastique analytique, assure la valeur des muscles dans l'exécution de la partition ludique et sportive. "(63)

En critiquant le sport spectacle, Tissié rejoint les nombreux pédagogues qui dénoncent à cette époque, auprès du ministre de l'Éducation nationale, les risques encourus dans les établissements scolaires. ${ }^{\left({ }^{(6)}\right)}$ Regrettant les déviations morales constatées lors des pratiques sportives, il s'insurge contre la généralisation d'un fâcheux état d'esprit chez les jeunes et l'usage de gros mots et autres jurons. ${ }^{(65)}$

Si l'attrait ludique du sport répond à une volonté de se divertir, Philippe Tissié lui préfere les jeux récréatifs, régis par des règles bien définies. Il estime en effet que « le sport est un provocateur de réflexes impulsifs d'ordre personnel » qui ne correspond pas au tempérament français ». ${ }^{\left({ }^{(6)}\right)}$ Pire, il peut constituer un danger pour la jeunesse qui, «mal conseillée et mal dirigée, en abuse avec ignorance ${ }^{(67)}$ Durant toute sa vie, il s'oppose à l'enseignement des sports violents, car ils provoquent, selon lui, non la santé mais la maladie. ${ }^{(68)}$ Léducation est donc au cœur de son projet de rééducation, car elle représente une démarche " réfléchie, prudente et opportune, d'action lente et douce, de respect de chaque sujet et de souci de son développement progressif par des sentiments affectifs d'ordre supérieur, où le cœur domine l'esprit, où la volonté est persuasive, où la solidarité faite d'estime réciproque est comme une appétence de l'âme au dévouement ". ${ }^{(6)}$ En développant la mầtrise de soi-même, le jeune délinquant peut quitter le monde de la criminalité pour devenir un "affirmatif " de mieux en mieux insérable dans une société démocratique. Cette conception sera finalement reconnue en 1938, lorsque ses contemporains lui rendront un hommage posthume en organisant à Pau le congrès mondial de culture humaine..$^{(70)}$

\section{Conclusion. Pour une génération de citoyens bons et beaux}

Si la prison a toujours poursuivi plusieurs objectifs, mettre hors d'état de nuire, punir par l'obligation d'une souffrance dont la durée et la modalité sont fonction du délit ou du crime, elle a toujours été aussi considérée comme un moyen de régénération de l'individu. ${ }^{(71)}$ Entre 1888 et 1938, Philippe Tissié tente de participer à cette double mission en proposant une « œuvre d'art et de

(65) Archives nationales, dossier F 1713 928, "Rapports des conseils d'académie de 1922 à 1924 sur l'enseignement secondaire public et privé, rapport sur l'enseignement secondaire des garçons dans l'académie de Poitiers pendant l'année scolaire 1921-1922 ", p. 5 bis.

(66) La Revue des jeux scolaires et d'hygiène sociale, 1933, p. 71-72.

(67) Philippe Tissié, «Grandeur et décadence de l'EP en France », $L a$ Revue des jeux scolaires et d'hygiène sociale, 1934, p. 27

(68) Philippe Tissié, L'éducation physique et la race..., op. cit., p. 136.

(69) Philippe Tissié, "Élevage et éducation ", op. cit., 1895, p. 17.

(70) Commandant Laulhé, « Méthode, technique et pédagogie en 
gymnastique éducative ", document manuscrit, archives de la Fédération française d'éducation physique et de gymnastique volontaire, document non référencé.

(71) Jacques-Guy Petit, Claude Faugeron, Michel Pierre, Histoire des prisons en France (I789-2000), Toulouse, Privat, 2002, $254 \mathrm{p}$.

(72) Philippe Tissié,

L'éducation physique et la race..., op. cit., p. 144.

(73 Ibid., p. 146.

(74) En 1919, il déclare notamment que « la gymnastique analytique aux mouvements disciplinés, vivifie, guérit et ne tue jamais ", ibid, p. 159. science en même temps qu'une œuvre pratique et nationale ». Patriote, Philippe Tissié se range aux côtés des médecins hygiénistes pour s'opposer au darwinisme social, en étant l'un des rares acteurs de l'éducation physique française à s'intéresser aux jeunes délinquants, dans le but de les rééduquer pour les ramener sur le droit chemin grâce à une éducation morale et corporelle empreinte de valeurs traditionnelles. Il serait donc bien candide de réduire sa mission éducatrice à un simple redressement du corps humain. Durant cinquante ans, Philippe Tissié tente de répondre à sa manière à un ensemble d'objectifs aux plans physique, psychique, moral et social, qui s'inscrivent toujours selon les circonstances dans leur actualité. Pour lui, la France est une « nation affective et affirmative ». Aux moments les plus critiques, le "Français se retrouve ce qu'il est vraiment, un affirmatif, que l'obstacle excite au lieu de rebuter, qui le rend plus fort, plus volontaire, plus patient ${ }^{(72)}$ :

«Un peuple n'est jamais vaincu tant qu'il est affirmatif, c'est-à-dire en puissance d'action. [...] Opposons la petite chèvre de M. Seguin aux moutons de Panurge. Le mouton passif, à l'œil morne, constitue les $75 \%$ de l'humanité ; la petite chèvre affirmative à l'œil vif, avec une expression de vie intelligente, constitue les $5 \%$ de l'humanité. Le mouton vit passivement en troupeau, par ordre serré, ainsi l'Allemand ; la chèvre, individualiste, vit affirmativement, en ordre dispersé, ainsi le Français capricant. " ${ }^{(73)}$

L'intérêt de Philippe Tissié pour les jeunes délinquants n'est donc pas surprenant. Au-delà du combat pour leur redressement biologique et social, il milite pour l'efficacité de la gymnastique franco-suédoise dans la régénération physique, psychologique et sociale des plus faibles. ${ }^{(74)}$ Seule importe en effet pour lui que la France ne devienne pas " un peuple paresseux et restrictif ». ${ }^{(75)}$ 